\title{
A GENERALIZATION OF THE DICHROMATIC POLYNOMIAL OF A GRAPH
}

\author{
E.J. FARRELL \\ Department of Mathematics \\ The University of the West Indies \\ St. Augustine, Trinidad \\ West Indies \\ (Received December 4, 1980)
}

ABSTRACT. The Subgraph polynomial fo a graph pair $(G, H)$, where $H \subseteq G$, is defined. By assigning particular weights to the variables, it is shown that this polynomial reduces to the dichromatic polynomial of $G$. This idea of a graph pair leads to a dual generalization of the dichromatic polynomial.

KEY WORDS AND PHRASES. Graph pair, cover of a graph, subgraph polynomial of a graph pair, reduction process, incorporated process, dichromatic polynomial of a graph pair, dichromate of a graph pair, chromatic polynomial of a graph pair.

1980 MATHEMATICS SUBJECT CLASSIFICATION CODES. 05A15, $05 C 99$.

1. INTRODUCTION.

The graphs considered here will be finite and undirected. Let $G$ be such a graph, and $\mathrm{H}$ a subgraph of $\mathrm{G}$. We define a graph pair to be an ordered pair (G,H). Let $\mathrm{K}$ be a spanning subgraph (or cover) of $\mathrm{G}$ containing $\mathrm{H}$. With every component $\alpha$ of $\mathrm{K}$, let us associate a weight $\mathrm{w}_{\alpha}$, and with $\mathrm{K}$, let us associate the weight

$$
\mathbf{w}(\mathrm{K})=\Pi \mathbf{w}_{\alpha},
$$

where the product is taken over all the component of $K$. Then the subgraph polynomial of the graph pair $(\mathrm{G}, \mathrm{H})$ is

$$
S((G, H): \underline{w})=\sum w(K),
$$

where the summation is taken over all the covers $K$ of $G$ such that $H \subseteq K \subseteq G$, and $\underline{w}$ is a vector of indetermainates associated with the given weights.

We will define the dichromatic polynomial, dichromate and chromatic polynomials of graph pairs and show that with approximately chosen weights, 
these polynomials reduce to their analogous well known counterparts.

\section{BASIC RESULTS}

We will say that an edge $e$ of $G$ is incorporated in $G$, if it is distinguished in some way and required to belong to every cover $K$ that we consider. An incorporated graph will be a graph whose edge set consists only of incorporated edges. Clearly then, $\mathrm{H}$ will be an incorporated graph when we consider the graph pair $(G, H)$. If the edge set of $H$ is empty, we will denote the graph pair $(G, H)$ by $G / H$. Of course, if $H$ is the null graph $(G, H)$ is essentially $G$ itself.

By putting the covers $\mathrm{K}$ into two classes according to whether or not they contain a specified unincorporated edge, we obtain the following theorem.

THEOREM 1. (The Fundamental Theorem). Let $\mathrm{G}$ be a graph containing an unincorporated edge e. Let $G^{\prime}$ be the graph obtained from $G$ by deleting $e$, and $H^{*}$ the graph obtained by adding the edge $e$ to $H$ - a subgraph of $G$; i.e. $E\left(H^{*}\right)=E(H) U\{e\}$. Then

$\mathrm{S}((\mathrm{G}, \mathrm{H}) ; \underline{w})=\mathrm{S}\left(\left(\mathrm{G}^{-}, \mathrm{H}\right) ; \underline{w}\right)+\mathrm{S}\left(\left(\mathrm{G}, \mathrm{H}^{*}\right) ; \underline{w}\right)$.

(Notice that Theorem 1 further generalizes the analogous result for F-polynomials given in Farrell [1]). By applying the theorem recursively, we can obtain an algorithm for finding subgraph polynomials of graph pairs. The incorporating process will depend on the criteria used for assigning weights to the covers. The implied algorithm will be called the fundamental algorithm for subgraph polynomials of graph pairs, or for brevity, the reduction process.

Let $G$ be a graph consisting of two components $G_{1}$ and $G_{2}$. Let $H$ be a subgraph of $G$ such that

$$
\text { (1) } E(H) \cap E\left(G_{1}\right)=E\left(H_{1}\right)
$$

and (ii) $E(H) \cap E\left(G_{2}\right)=E\left(H_{2}\right)$,

where $\mathrm{H}_{1}$ and $\mathrm{H}_{2}$ are subgraphs of $\mathrm{H}$. Then every cover of $\mathrm{G}$ can be broken up into a cover of $G_{1}$ containing $H_{1}$ and cover of $G_{2}$ containing $H_{2}$. Conversely, every cover of $G_{1}$ containing $H_{1}$ can be combined with any cover of $G_{2}$ containing $H_{2}$, to yield a cover of $G$. By generalizing this discussion we can prove the following theorem.

THEOREM 2. If $G$ consists of components $G_{1}, G_{2}, \ldots, G_{n}$, then 


$$
S((G, H) ; \underline{w})=\prod_{i=1}^{n} S\left(\left(G_{i}, H_{i}\right): \underline{w}\right),
$$

where $H_{i}$ are respectively subgraphs of $G_{i}$ such that $E(H) \cap E\left(G_{i}\right)=E\left(H_{i}\right)$ $(i=1,2, \ldots, n)$.

(Notice that it is possible to have $\left(G_{i}, H_{i}\right)$ isomorphic as a graph pair to $\left(G_{j}, H_{j}\right)$, while $S\left(\left(G_{i}, H_{i}\right) ; \underline{w}\right) \neq S\left(\left(G_{j}, H_{j}\right) ; \underline{w}\right)$, since our definition allows the possibility that isomorphic components of $G$ be given different weights).

\section{APPLICATION TO DICHROMATIC POLYNOMIALS}

Let us put $\underline{w}=\left(w_{i j}: i \geq 1, j \geq 0\right)$, where $i$ and $j$ are the number of nodes and edges respectively in the component. Then the subgraph polynomial of $(G, H)$ can be written as

$$
S((G, H) ; \underline{w})={ }_{H \subseteq K \subseteq G} \Pi w_{i j}^{n_{i j}},
$$

where $n_{i j}$ is the number of components of $K$ that contain $i$ nodes and $j$ edges. By putting $w_{i j}=x y^{j-i+1}$, we get the polynomial

$$
\begin{aligned}
& Q((G, H) ; x, y)=\sum_{H \subseteq K \subseteq G} \Pi\left(x y^{j-i+1)^{n i j}}\right. \\
&={ }_{H} \subseteq K \subseteq G \\
& x^{c} y^{a-b+c},
\end{aligned}
$$

where $c=\sum_{i, j} n_{i j}$ is the number of components in $k$,

$$
a=\sum_{i, j} j n_{i j} \text { is the number of edges in } k \text {, }
$$

and $\quad b=\sum_{i, j} i n_{i j}$ is the number of nodes in $k$.

The polynomial of $Q((G, H) ; \underline{w})$ will be called the dichromatic polynomial of the graph pair $(G, H)$, or the Tutte polynomial of $(G, H)$. It is clear that if $H$ is empty, the polynomial $Q(G / H ; x, y)$ is the dichromatic polynomial of $G$ as defined in Tutte [2]. Thus we have the following result.

THEOREM 3. The polynomial obtained from $S(G / H ; \underline{w})$ by putting $\underline{w}=\left(w_{i j}: i \geq 1, j \geq 0\right)$, with $w_{i j}=x y^{j-i+1}$, where $i$ and $j$ are the number of nodes and edges respectively in the component, is the dichromatic polynomial of $\mathrm{G}$. 
The polynomial $Q((G, H) ; x, y)$ gives a dual generalization of the dichromatic polynomial of a graph, since

(i) the polynomial can be taken relative to a fixed subgraph and

(ii) different weights can be attached to subgraphs.

Let us put $w_{i j}=[(x-1)(y-1)]^{1-i}(y-1)^{j}$ in Equation (1), and let $p$ be the number of nodes in $G$. Then we get the polynomial

$$
\begin{aligned}
R((G, H) ; x, y) & =\sum_{H \subseteq K \subseteq G} \Pi\left\{[(x-1)(y-1)]^{1-1}(y-1)^{j}\right\}^{n_{i j}} \\
& =\sum(x-1)^{-p}(x-1)^{c}(y-1)^{a-b+c} \\
& =(x-1)^{-p} \sum(x-1)^{c}(y-1)^{a-b+c} \\
& =(x-1)^{-p} Q((G, H) ; x-1, y-1) .
\end{aligned}
$$

We define $R((G, H): x, y)$ to be the dichromate of the graph pair $(G, H)$.

Equation (2) shows an analogous relation between the dichromate and dichromatic polynomials of a graph pair.

The following result is clear from the definition of the dichromate of a graph given in [2].

THEOREM 4. The polynomial obtained from $\mathrm{S}(\mathrm{G} / \mathrm{H} ; \underline{w})$ by putting $\underline{w}=\left(w_{i j}: i \geq 1, j \geq 0\right)$, with $w_{i j}=[(x-1)(y-1)]^{1-i}(y-1)^{j}$, where $i$ and $j$ are the number of nodes and edges respectively in the component, is the dichromate of $\mathrm{G}$.

\section{APPLICATION TO CHROMATIC POLYNOMIALS}

Let us put $\underline{w}=\left(w_{i}: i \geq 0\right)$, where $i$ is the number of edges in the component. Then the subgraph polynomial of $(G, H)$ (where $G$ is a graph with $p$ nodes and q edges) becomes

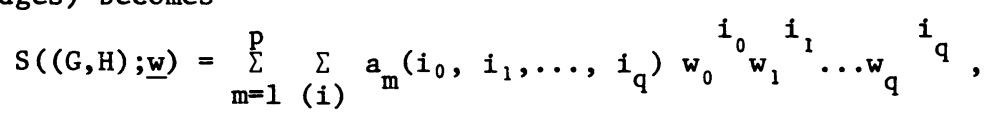

where $a_{m}\left(i_{0} i_{1}, \ldots, i_{q}\right)$ is the number of covers which contain $H$, and have $m$ components consisting of $i_{0}$ isolated nodes, $i_{1}$ edges, $i_{2}$ components with 2 edges etc., and the second summation is taken over all solutions of $\sum_{r=0}^{q} i_{r}=m$.

Specifically, if we put $w_{i}=(-1)^{i} \lambda$, then Equation (3) yields the following polynomial.

$$
P((G, H):)=\sum_{m=1}^{p} \sum_{(i)} a_{m}\left(i_{0}, i_{1}, \ldots, i_{q}\right)(-1)^{n} \lambda^{m},
$$


where $n=\sum_{j=1}^{q} j i_{j}$. Since all components with the same number of edges will receive the same weight, we can replace $a_{m}\left(i_{0}, i_{1}, \ldots, i_{q}\right)$ by $a_{m}(n)-$ the number of covers of $\mathrm{G}$ containing $\mathrm{m}$ components and $\mathrm{n}$ edges. Thus Equation (4) becomes

$$
\begin{aligned}
P((G, H) ; \lambda) & =\sum_{m=1}^{p}\left[\sum_{n=0}^{q}(-1)^{n} a_{m}(n)\right] \lambda^{m} \\
& =\sum_{m=1}^{p} d_{m} \lambda^{m},
\end{aligned}
$$

where $d_{m}=\sum_{n=0}^{q}(-1)^{n} a_{m}(n)$. The polynomial $P((G, H) ; \lambda)$ will be called the chromatic polynomial of the graph pair $(G, H)$.

The following result is clear from the material above, and the definition of the chromatic polynomial of a graph given in whitney [3].

THEOREM 5. The polynomial obtained from $S(G / H ; \underline{w})$ by putting $\underline{w}=\left(w_{i}: i \geq 0\right)$, with $w_{i}=(-1)^{i} \lambda$, where $i$ is the number of edges in the component, is the chromatic polynomial of $\mathrm{G}$.

By establishing a relation between $x y^{j-i+1}$ and $(-1)^{j} \lambda$, we obtain the following result.

THEOREM 6. $P((G, H) ; \lambda)=(-1)^{P} Q((G, H) ;-\lambda,-1)=\lambda^{P} R((G, H) ; 1-\lambda, 0)$.

\section{REFERENCES}

1. FARRELL, E.J. On a general class of graph polynomials, J. Comb. Theory B 26 (1979), $111-122$.

2. TUTTE, W.T. The dichromatic polynomial, Proc. of the Fifth British Comb. Conf. (1975), $605-635$.

3. WHITNEY, H. A logical expansion in Mathematics, Bull.Amer. Math. Soc. 38 (1932), $572-579$. 


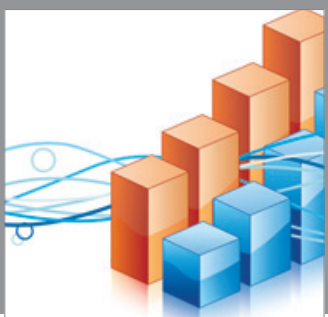

Advances in

Operations Research

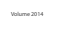

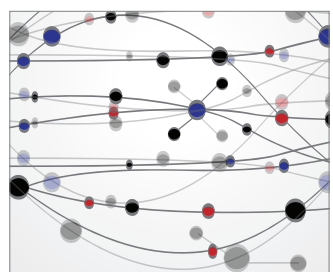

\section{The Scientific} World Journal
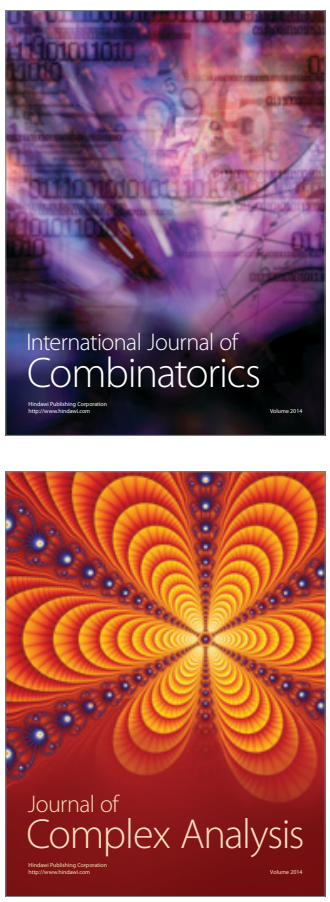

International Journal of

Mathematics and

Mathematical

Sciences
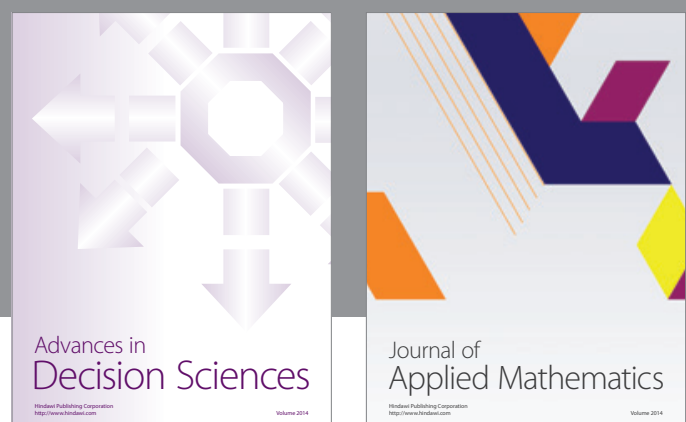

Journal of

Applied Mathematics
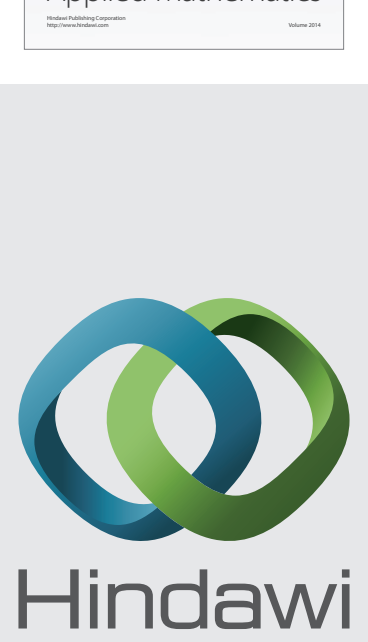

Submit your manuscripts at http://www.hindawi.com
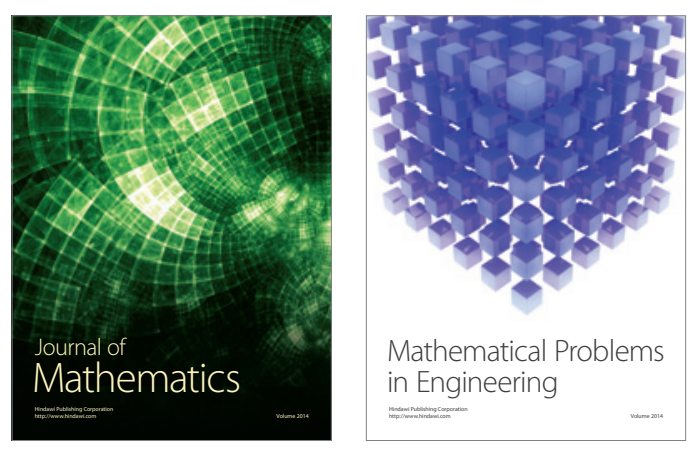

Mathematical Problems in Engineering
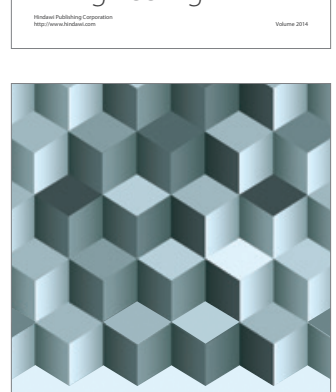

Journal of

Function Spaces
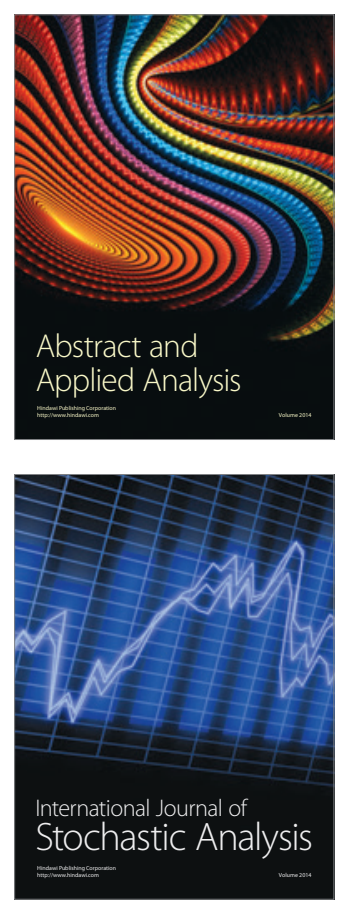

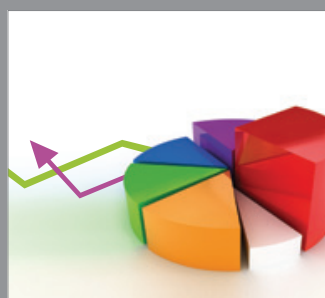

ournal of

Probability and Statistics

Promensencen
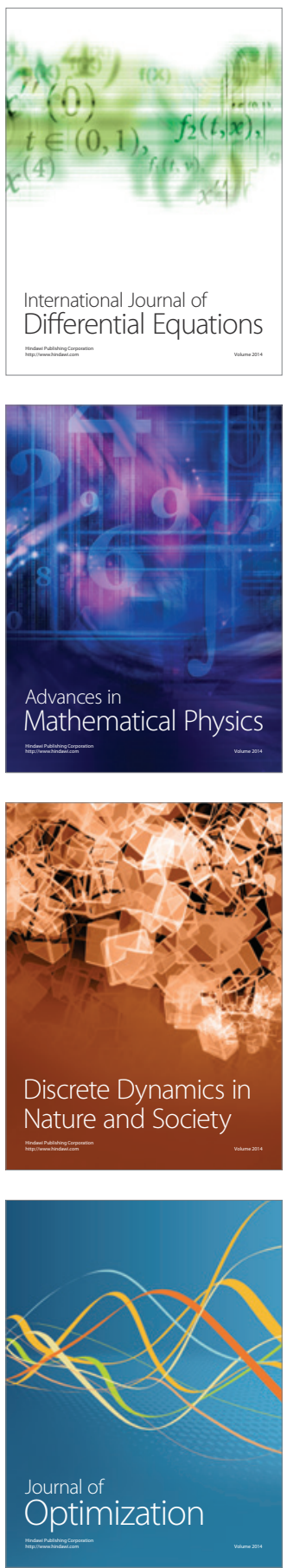